\title{
Deterministic BIST Based on a Reconfigurable Interconnection Network $^{\star}$
}

\author{
Lei Li and Krishnendu Chakrabarty \\ Department of Electrical and Computer Engineering \\ Duke Universit\}, Durham, NC 27708 \\ 1l,krish@ee.duke.edu
}

\begin{abstract}
We present a new approach for deterministic BIST in which a reconfigurable interconnection network (RIN) is placed between the outputs of a pseudo-random pattern generator and the scan inputs of the circuit under test (CUT). The RIN, which consists only of multiplexer switches, replaces the phase shifter that is typically used in pseudo-random BIST to reduce correlation between the test data bits that are fed into the scan chains. The connections between the LFSR and the scan chains can be dynamically changed (reconfigured) during a test session. In this way, the RIN is used to match the LFSR outputs to the test cubes in a deterministic test set. The control data bits used for reconfiguration ensure that all the deterministic test cubes are embedded in the test patterns applied to the CUT. The proposed approach requires very little hardware overhead, and fewer control bits compared to the storage required for reseeding techniques or for hybrid BIST. Moreover, as a non-intrusive BIST solution, it does not require any circuit redesign and has minimal impact on circuit performance.
\end{abstract}

\section{INTRODUCTION}

Higher circuit densities and ever-increasing design complexity are placing a severe burden on the automatic test equipment (ATE) used to test today's integrated circuits (ICs). The integration of complex embedded cores in system-on-a-chip (SOC) designs is leading to a sharp increase in test data volume, which requires significant investment in additional memory depth per ATE channel. ATE channel bandwidth is another limitation for SOCs with high clock frequencies, enormous test data volume, and a large number of I/O pins. In order to mitigate these problems, a number of techniques based on test data compression, built-in self-test (BIST), and a combination of the two have been proposed in the literature.

In the test data compression approach, a deterministic test set is compressed and stored in ATE memory. The compressed test set is transferred through ATE channels to the IC, where it is decompressed and applied to the circuit under test (CUT) by decoding hardware. Techniques based on statistical coding [12, 14], run-length coding [13], Golomb coding [5], FDR coding [4], and VIHC coding [8], have been proposed to reduce test data volume. Test data volume reduction techniques

\footnotetext{
* This research was supported in part by the National Science Foundation under grants CCR-9875324 and CCR-0204077, and by a graduate fellowship from the Design Automation Conference.
}

based on on-chip pattern decompression are also presented in $[3,7,19,24,25,26,29]$.

The resurgence of interest in test data compression has also led to new commercial tools that can provide substantial compression for large industrial designs. For example, the OPMISR [2] and SmartBIST [16] tools from IBM and the TestKompress tool from Mentor Graphics [23] reduce test data volume and testing time through the use of test data compression and on-chip decompression.

In BIST solutions, test patterns are generated by an onchip pseudo-random pattern generator, which is usually a linear-feedback shift-register (LFSR). BIST alleviates a number of problems related to test interfacing, e.g., limited signal bandwidth, high pin count. A typical BIST architecture is shown in Fig. 1. In order to detect the random-patternresistant faults and achieve complete coverage of single stuckat faults, techniques based on test point insertion [6, 27], reseeding [10, 20, 22], bit-flipping [31], bit-fixing [28] and weighted random pattern testing [30] have been proposed. Test point insertion techniques require design changes to improve random pattern testability such that $100 \%$ fault coverage can be achieved using a reasonable number of pseudo-random test patterns. The other BIST techniques are non-intrusive in that they typically apply a limited number of random patterns, then for the remaining hard to test faults, deterministic test patterns are obtained by either controlling the state of the pattern generator $[10,17,20,22]$ or by altering the output of the pattern generator [28, 30, 31]. A number of studies have also been reported recently on the use of BIST for large industrial circuits $[11,21]$.

Techniques based on the combination of data compression and BIST have also been developed recently $[15,18]$. The hybrid BIST scheme presented in [15] applies weighted pseudorandom patterns to the circuit to achieve $100 \%$ fault coverage. The compressed weight set is stored on ATE and decompression is carried out using an on-chip look-up table. In [18], the seeds for the LFSR are compressed using statistical coding.

In this paper, we present a new deterministic BIST approach in which a reconfigurable interconnection network (RIN) is placed between the outputs of the LFSR and the inputs of the scan chains in the CUT. The RIN, which consists only of multiplexer switches, replaces the phase shifter that is typically used 


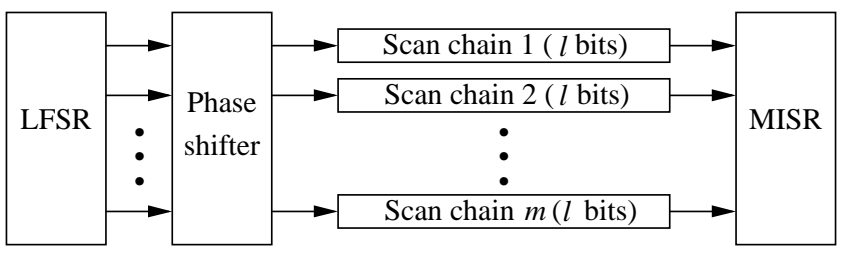

Fig. 1. A generic BIST architecture based on an LFSR, a MISR, and a phase shifter.

in pseudo-random BIST to reduce correlation between the test data bits that are fed into the scan chains. The connections between the LFSR and the scan chains can be dynamically changed (reconfigured) during a test session. In this way, the RIN is used to match the LFSR outputs to the test cubes in a deterministic test set. The control data bits used for reconfiguration ensure that all the deterministic test cubes are embedded in the test patterns applied to the CUT. The proposed approach requires very little hardware overhead, and fewer control bits compared to the storage required for reseeding techniques or for hybrid BIST. Moreover, as a non-intrusive BIST solution, it does not require any circuit redesign and has minimal impact on circuit performance.

The rest of the paper is organized as follows. Section II presents an overview of related prior work. In Section III, we present the architecture of the proposed BIST scheme and describe the procedure for the synthesis of the reconfigurable interconnection network and the determination of the control bits. In Section IV, we describe a strategy for declustering the care bits in the test cubes to improve the efficiency of the proposed method. Experimental results and a comparison with related recent work are presented in Section V. Finally, Section VI concludes the paper.

\section{RELATED PRIOR WORK}

Most BIST techniques rely on the use of a limited number of pseudo-random patterns to detect the random-patterntestable faults, which is subsequently followed by the application of a limited number of deterministic patterns to detect the random-pattern-resistant faults. Based on the mechanisms that are used to generate the deterministic patterns, BIST techniques can be classified into two categories: methods that generate deterministic patterns by controlling the states of the LFSR $[10,17,20,22]$, and techniques that modify the patterns generated by the LFSR [28, 30, 31].

LFSR reseeding is an example of a BIST technique that is based on controlling the LFSR state. LFSR reseeding can be static, i.e., the LFSR stops generating patterns while loading seeds, or dynamic, i.e., test generation and seed loading can proceed simultaneously. The length of the seeds can be either equal to the size of the LFSR (full reseeding) or less than the size of the LFSR (partial reseeding). In [17], a dynamic reseeding technique that allows partial reseeding is proposed to encode test vectors. An LFSR of length $r \geq s_{\max }+20$, where $s_{\max }$ is the maximum number of specified bits in any deterministic test cube, is used to generate the test patterns. While the length of the first seed is $r$, the lengths of the subsequent seeds are significantly smaller than $r$. A set of linear equations is solved to obtain the seeds, and the test vectors are reordered to facilitate the solution of this set of linear equations.

A BIST pattern generator based on a folding counter is proposed in [10]. The properties of the folding counter are exploited to find the seeds needed to cover the given set of deterministic patterns. Width compression is combined with reseeding to reduce the hardware overhead. In [20], a twodimensional test data compression technique that combines an LFSR and a folding counter is proposed for scan-based BIST. LFSR reseeding is used to reduce the number of bits to be stored for each pattern (horizontal compression) and folding counter reseeding is used to reduce the number of patterns (vertical compression).

Bit-flipping, bit-fixing and weighted random BIST are examples of techniques that rely on altering the patterns generated by the LFSR to embed deterministic test cubes. In [15], a hybrid BIST method based on weighted pseudo-random testing is presented. A weight of 0,1 or $\mathrm{u}$ (unbiased) is assigned to each scan chain in CUT. The weight sets are compressed and stored on the tester. During test application, an on-chip look-up table is used to decompress the data from the tester and generate the weight sets. A 3-weight weighted random scan-BIST scheme is discussed in [30]. The weights in this approach are $0,0.5$, and 1 . In order to reduce the hardware overhead, scan cells are carefully reordered and a special ATPG approach is used to generate suitable test cubes.

\section{PROPOSED APPROACH}

In a generic LFSR-based BIST approach shown in Fig. 1, the output of the LFSR is fed to a phase shifter to reduce the linear dependency between the data shifted into different scan chains. The phase shifter is usually a linear network composed of exclusive-or gates. In the proposed approach, illustrated in Fig. 2(a), the phase shifter is replaced by a reconfigurable interconnection network (RIN) that connects the LFSR outputs to the scan chains. The RIN consists of multiplexer switches and it can be reconfigured by applying appropriate control bits to it through the inputs $D_{0}, D_{1}, \ldots, D_{g-1}$. The parameter $g$ refers to the number of configurations used during a BIST session and it is determined using a simulation procedure. The control inputs $D_{0}, D_{1}, \ldots, D_{g-1}$ are provided by a $d$-to- $g$ decoder, where $d=\left\lceil\log _{2} g\right\rceil$. A $d$-bit configuration counter is used to cycle through all possible $2^{d}$ input combinations for the decoder. The configuration counter is triggered by the BIST pattern counter, which is preset for each configuration by the binary value corresponding to the number of test patterns for the corresponding configuration.

As shown in Fig. 2(b), the multiplexers in the RIN are implemented using tristate buffers with fully-decoded control inputs. While the multiplexers can also be implemented in other ways, 


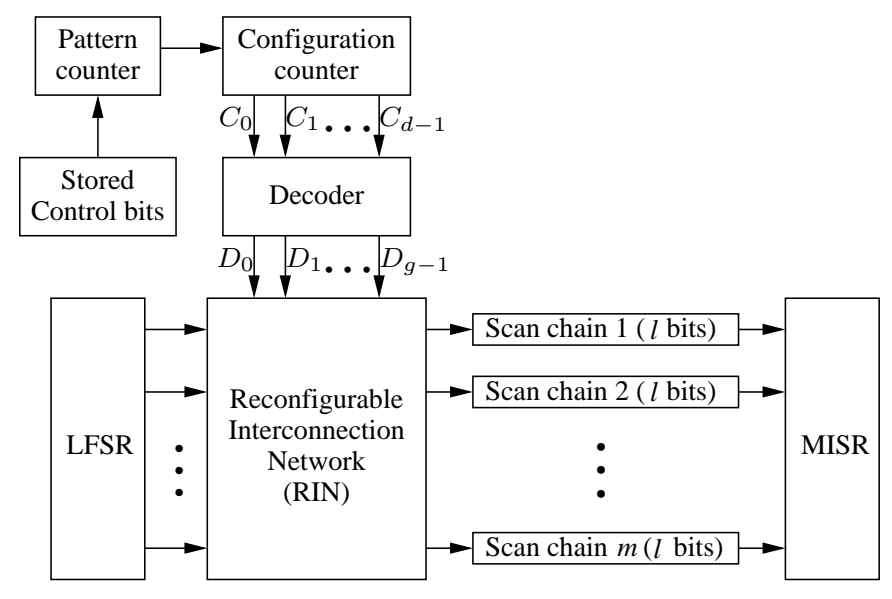

( a )

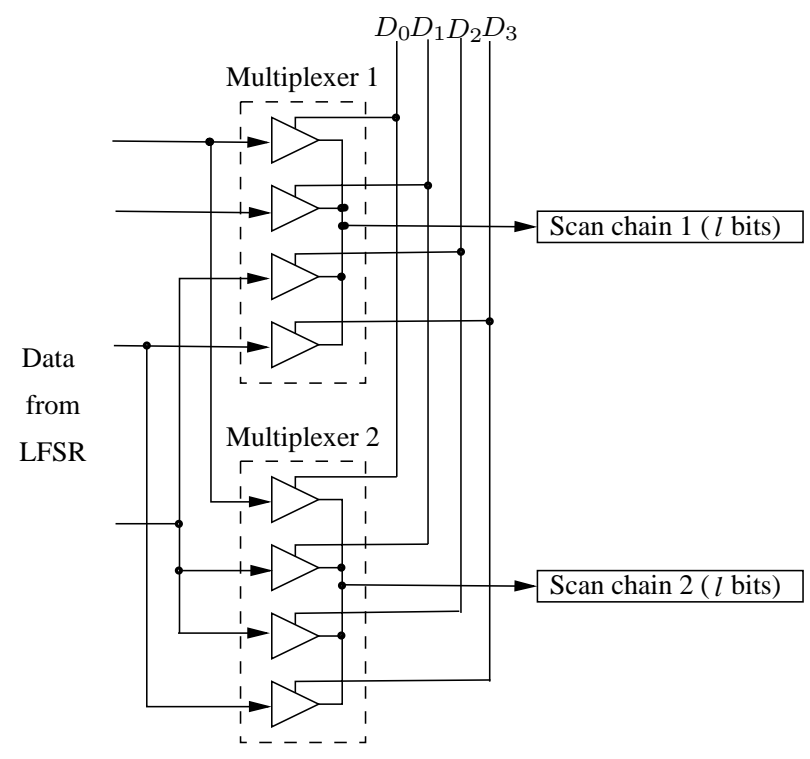

( b )

Fig. 2. (a) Proposed BIST architecture (b) Reconfigurable interconnection network for $m=2$ and $g=4$.

we use tristate buffers here because of ease of implementation in CMOS. The outputs of the tristate buffers are connected at the output of the multiplexer. Each input $I_{i}$ of a multiplexer is connected to the input of a tristate buffer, which is controlled by the corresponding control signal. While the number of multiplexers equals the number of scan chains, the number of tristate gates in each multiplexer is equal to the number of configurations.

We next describe the test application procedure during a BIST session. First the configuration counter is reset to the all-0 pattern, and the pattern counter is loaded with the binary value corresponding to the number of patterns that must be applied to the CUT in the first configuration. The pattern counter is decremented each time a test pattern is applied to the

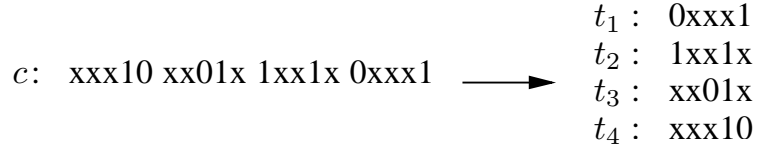

Fig. 3. A illustration of converting a test cube to multiple scan chain format $(m=4, l=5)$.

CUT. When the content of the pattern counter become zero, it is loaded with the number of patterns for the second configuration and it triggers the configuration counter, which is incremented. This leads to a corresponding change in the outputs of the decoder, and the RIN is reconfigured appropriately. This process continues until the configuration counter passes through all $g$ configurations. The total number of test patterns applied to the CUT is therefore $\sum_{i=1}^{g} n_{i}$, where $n_{i}$ is the number of patterns corresponding to configuration $i, 1 \leq i \leq g$. The BIST design procedure described next is tailored to embed a given set of deterministic test cubes in the sequence of $\sum_{i=1}^{g} n_{i}$ patterns.

During test application, pseudo-random patterns that do not match any deterministic test cube are also applied to the CUT. These pseudo-random patterns can potentially detect non-modeled faults. However, these patterns increase the testing time. A parameter called MaxSkipPatterns, which is defined as the largest number of pseudo-random patterns that are allowed between the matching of two deterministic cubes, is used in the design procedure to limit the testing time. We first need to determine for each configuration, the number of patterns and the interconnections between the LFSR outputs and the scan chains. We use a simulation procedure to solve this problem. We start with an LFSR of length $L$, a predetermined seed, and a known characteristic polynomial. Let $T_{D}=\left\{c_{1}, c_{2}, \ldots, c_{n}\right\}$ be the set of deterministic test cubes that must be applied to the CUT. The set $T_{D}$ can either target all the single stuck-at faults in the circuit, or only the hard faults that cannot be detected by a small number of pseudo-random patterns. As illustrated in Fig. 3, each deterministic test cube $c$ in the test set is converted into the multiple scan chain format as a set of $m l$-bit vectors $\left\{t_{1}, t_{2}, \ldots, t_{m}\right\}$, where $m$ is the number of scan chains and $l$ is the length of each scan chain. The bits in a test cube are ordered such that the least significant bit is first shifted into the scan chain. We use $\operatorname{Conn}_{j}^{(i)}$ to denote the set of LFSR taps that are connected to the scan chain $j$ in configuration $i$, where $i=1,2, \ldots, g, j=1,2, \ldots, m$. The simulation procedure is as follows.

1) Set $i=1$.

2) Set $\mathrm{Conn}_{j}^{(i)}=\{1,2, \ldots, L\}$ for $j=1,2, \ldots, m$, i.e., initially, each scan chain can be connected to any tap of the LFSR.

3) Driving the LFSR for the next $l$ clock cycles, we obtain the output of the LFSR as a set of $L l$-bit vectors $\left\{O_{k} \mid k=1,2, \ldots, L\right\}$, where vector $O_{k}$ is the output stream of the $k$ th flip-flop of the LFSR for the $l$ clock cycles. 
4) Find a test cube $c^{*}$ in $T_{D}$ that is compatible with the outputs of the LFSR under the current connection configuration $\operatorname{Conn}_{j}^{(i)}$, i.e., for all $j=1, \ldots, m$, there exists $k \in$ Conn $_{j}^{(i)}$ such that $t_{j}^{*}$ is compatible with $O_{k}$, where $c^{*}$ has already been reformatted for $m$ scan chains as a set of vector $\left\{t_{1}^{*}, t_{2}^{*}, \ldots, t_{m}^{*}\right\}$. ( A vector $u_{1}, u_{2}, \ldots, u_{r}$ and a vector $v_{1}, v_{2}, \ldots, v_{r}$ are mutually compatible if for any $i, 1 \leq i \leq r$, one of the following holds: (i) $u_{i}=v_{i}$ if they are both care bits; (ii) $u_{i}$ is a don't-care bit; (iii) $v_{i}$ is a don't-care bit.)

5) If no test cube is found in Step 4, go to Step 6 directly. Otherwise, remove the test cube $c^{*}$ found in Step 4 from $T_{D}$, and narrow down the connection configuration as follows. For each $j=1,2, \ldots, m$, let $\mathbf{U} \subset$ Conn $_{j}^{(i)}$ such that for any $k \in \mathbf{U}, O_{k}$ is not compatible with $t_{j}^{*}$. Then set $\operatorname{Conn}_{j}^{(i)}=\operatorname{Conn}_{j}^{(i)}-\mathbf{U}$.

6) If in the previous MaxSkipPatterns +1 iterations, at least one test cube is found in Step 4, then go to Step 3. Otherwise, the simulation for the current configuration is concluded. The patterns that are applied to the circuit under this configuration are those that are obtained in Step 3.

7) Match the remaining cubes in $T_{D}$ to the test patterns for the current configuration, i.e., if any test vector in $T_{D}$ is compatible with any pattern for the current configuration, remove it from $T_{D}$.

8) If no pseudo-random pattern for the current configuration is compatible with a test cube, the procedure fails and exits. Otherwise, increase $i$ by 1 , and go to Step 2 to begin the iteration for the next configuration until $T_{D}$ is empty.

An example of the simulation procedure is illustrated in Fig. 4. A 4-bit autonomous LFSR with characteristic polynomial $x^{4}+x+1$ is used to generate the pseudo-random patterns. There are four scan chains and the length of each scan chain is 4 bits. The parameter MaxSkipPatterns is set to 1 . The output of the LFSR is divided into patterns $p_{i}, i=1,2, \ldots$. Each pattern consists of four 4-bit vectors. The procedure that determines the connections is shown as Step Init) to Step f). Step Init) is the initialization step in which all the connections $\operatorname{Conn}_{j}^{(1)}, j=1,2,3,4$ are set to $\{1,2,3,4\}$. In Step a), the first pattern $p_{1}$ is matched with the test cube $c_{1}$, and the connections is shown for each scan chain: Scan chain 1 can be connected to $x_{1}$ or $x_{4}$, both Scan chain 2 and Scan chain 3 can only be connected to $x_{2}$, Scan chain 4 can be connected to $x_{1}, x_{2}$ or $x_{4}$. In Step c), none of the cubes is compatible with $p_{3}$. When neither $p_{5}$ nor $p_{6}$ matches any cubes in Step e), the iterations for the current configuration are terminated. The patterns that are applied to the CUT in this configuration is $p_{1}, p_{2}, \ldots, p_{6}$. We then compare the remaining cube $c_{4}$ with the six patterns and find that it is compatible with $p_{2}$. So $c_{4}$ is also covered by the test patterns for the current configuration. Thus the connections for this configuration are: Scan chain 1 is

\section{LFSR:}

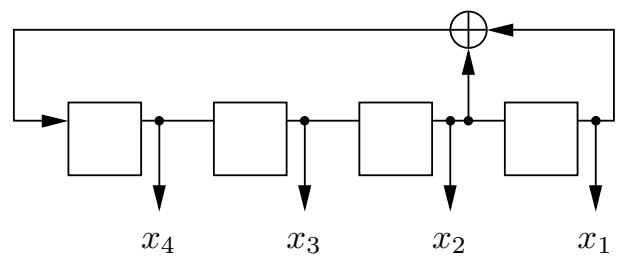

Output of LFSR:

$\begin{array}{ccccccccc} & & \ldots & p_{6} & p_{5} & p_{4} & p_{3} & p_{2} & p_{1} \\ x_{1} & : & \ldots & 1100 & 1000 & 1111 & 0101 & 1001 & 0001 \\ x_{2} & : & \ldots & 0110 & 0100 & 0111 & 1010 & 1100 & 1000 \\ x_{3} & : & \ldots & 1011 & 0010 & 0011 & 1101 & 0110 & 0100 \\ x_{4} & : & \ldots & 0101 & 1001 & 0001 & 1110 & 1011 & 0010\end{array}$

Test cubes:

$\begin{array}{cccc}c_{1} & c_{2} & c_{3} & c_{4} \\ 00 \mathrm{xx} & 0 \mathrm{xxx} & \mathrm{xx} 11 & \mathrm{xx} 11 \\ 1 \mathrm{xx} 0 & \mathrm{xx} 1 \mathrm{x} & \mathrm{x} 10 \mathrm{x} & 1 \mathrm{xxx} \\ 10 \mathrm{xx} & 01 \mathrm{xx} & \mathrm{x} 1 \mathrm{x} 0 & 01 \mathrm{xx} \\ \mathrm{x} 0 \mathrm{xx} & 11 \mathrm{xx} & 10 \mathrm{xx} & \mathrm{x} 001\end{array}$

Determination of connections:

\begin{tabular}{|c|c|c|c|}
\hline \multicolumn{2}{|c|}{ Init) } & a) $p_{1}: c_{1}$ & b) $p_{2}: c_{3}$ \\
\hline Conn $_{1}^{(1)}:$ & $(1,2,3,4)$ & $(1,4)$ & (4) \\
\hline $\operatorname{Conn}_{2}^{(1)}$ : & $(1,2,3,4)$ & (2) & (2) \\
\hline $\operatorname{Conn}_{3}^{(1)}:$ & $(1,2,3,4)$ & (2) & (2) \\
\hline Conn $_{4}^{(1)}:$ & $(1,2,3,4)$ & $(1,2,4)$ & $(1,4)$ \\
\hline c) $p_{3}:$ none & d) $p_{4}: c_{2}$ & e) $p_{5}, p_{6}:$ none & f) $p_{2}: c_{4}$ \\
\hline (4) & (4) & (4) & (4) \\
\hline (2) & (2) & (2) & (2) \\
\hline (2) & (2) & (2) & (2) \\
\hline$(1,4)$ & (1) & (1) & (1) \\
\hline
\end{tabular}

Fig. 4. An illustration of the simulation procedure.

connected to $x_{4}$, both Scan chain 2 and 3 are connected to $x_{2}$, Scan chain 4 is connected to $x_{1}$. Since $p_{5}$ and $p_{6}$ are not compatible with any deterministic cubes, the number of patterns for this configuration is set to four. If there are test cubes remaining to be matched, the iteration for the next configuration starts from $p_{5}$.

\section{DeClustering THE CARE BITS}

The simulation procedure to determine the number of patterns and the connections for each configuration can sometimes fail to embed the test cubes in the LFSR sequence. This can happen if MaxSkipPatterns is too small, or the test cubes are hard to match with the outputs of the LFSR. During 
our experiments, we found that it was very difficult to embed the test cubes for the s38417 benchmark circuit. On close inspection, we found that the care bits in some of the test cubes for s38417 are highly clustered, even though the percentage of care bits in $T_{D}$ is small. When these test cubes are converted into a multiple scan chain format, most of the vectors contain very few care bits but a few vectors contain a large number of care bits. These vectors with many care bits are hard to embed in the output sequence of the LFSR.

In order to embed test cubes with highly clustered care bits, we propose two declustering strategies. The first is to reorganize the scan chains such that the care bits can be scattered across many scan chains, and each scan chain contains only a few care bits. Another strategy is based on the use of additional logic to interleave the data that are shifted into the different scan chains. The first strategy requires reorganization of the scan chains, but it does not require extra hardware overhead. The interleaving method does not modify the scan chains, but it requires additional hardware.

The method of reorganization of scan chains is illustrated in Fig. 5. As shown in the figure, before the reorganization, all the care bits of the given test cube are grouped in the second vector, which is hard to match with the output of LFSR. After the reorganization, the care bits are scattered across all the vectors, and the largest number of care bits in a vector is only two. This greatly increases the probability that this vector can be matched to an output pattern of the LFSR. Note that the concept of reorganization of scan chains is also used in [10]. However, the reorganization used in [10] changes the scan chain structure and makes it unsuitable for response capture - a separate solution is needed in [10] to circumvent this problem. In our approach, the basic structure of the scan chains is maintained and the usual scan test procedure of pattern shift-in, response capture, and shift-out can be used.

The scan cells in the CUT can be indexed as $c_{i, j}, i=$ $0,1, \ldots, m-1, j=0,1, \ldots, l-1$, where $m$ is the number of scan chains and $l$ is the length of a scan chain. Note that we start the indices from 0 to facilitate the description of the scan chain reorganization procedure. The $i$ th scan chain consists of the $l$ scan cells $c_{i, j}, j=0,1, \ldots, l-1$. We use $c_{i, j}^{*}$ to denote the reorganized scan cells, in which the $i$ th scan chain consists of the $l$ scan cells $c_{i, j}^{*}, j=0,1, \ldots, l-1$. For each $j=0,1, \ldots, l-1$, the $m$ cells $c_{0, j}, c_{1, j}, \ldots, c_{m-1, j}$ constitute a vertical vector. The reorganized scan cell structure is obtained by rotating each such vertical vector upwards by $d$ positions, where $d=j \bmod m$, i.e., $c_{i, j}^{*}=c_{k, j}$, where $k$ is given by $k=(i+d) \bmod m$.

An alternative method for declustering, based on the interleaving of the inputs to the scan chains, is shown in Fig. 6. We insert an extra stage of multiplexers between the outputs of the RIN and the inputs of the scan chains. From the perspective of the RIN, the logic that follows it, i.e., the combination of the multiplexers for interleaving and the scan chains, is simply a reorganized scan chain with an appropriate arrangement of
Test cube : $\mathrm{x} \times \mathrm{x} \times \mathrm{x} \times \mathrm{x} \times \mathrm{x} \times \mathrm{x} \times \mathrm{x} \times \mathrm{x} \times \mathrm{x} \times \mathrm{x} 100110 \mathrm{x} \times \mathrm{x} \times \mathrm{x} \times$ Reformat

Vectors

Scan cells

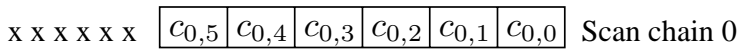

\begin{tabular}{lllll|l|l|l|l|l|l|l|}
100 & 0 & 0 & $c_{1,5}$ & $c_{1,4}$ & $c_{1,3}$ & $c_{1,2}$ & $c_{1,1}$ & $c_{1,0}$ & Scan chain 1
\end{tabular}

\begin{tabular}{ll|l|l|l|l|l|l} 
x x x x x x & $c_{2,5}$ & $c_{2,4}$ & $c_{2,3}$ & $c_{2,2}$ & $c_{2,1}$ & $c_{2,0}$ \\
\hline
\end{tabular}

\begin{tabular}{l|l|l|l|l|l|l|l}
$\mathrm{x} \times \mathrm{x} \times \mathrm{x} \times \mathrm{x}$ & $c_{3,5}$ & $c_{3,4}$ & $c_{3,3}$ & $c_{3,2}$ & $c_{3,1}$ & $c_{3,0}$ \\
\hline
\end{tabular}

\begin{tabular}{l|l|l|l|l|l|l|l} 
x x x x x x & $c_{4,5}$ & $c_{4,4}$ & $c_{4,3}$ & $c_{4,2}$ & $c_{4,1}$ & $c_{4,0}$ \\
\hline
\end{tabular}

$\sqrt{ }$ Reorganization $]$

\begin{tabular}{ll|l|l|l|l|l|}
$\mathrm{x} \times \mathrm{x} \times \mathrm{x} 1 \mathrm{x}$ & $c_{0,5}$ & $c_{4,4}$ & $c_{3,3}$ & $c_{2,2}$ & $c_{1,1}$ & $c_{0,0}$ \\
\hline
\end{tabular}

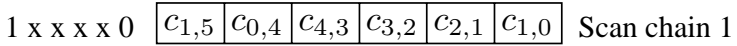

\begin{tabular}{l|l|l|l|l|l|l|l}
$\mathrm{x} 0 \times \mathrm{x} \times \mathrm{x}$ & $c_{2,5}$ & $c_{1,4}$ & $c_{0,3}$ & $c_{4,2}$ & $c_{3,1}$ & $c_{2,0}$ \\
\hline
\end{tabular}

\begin{tabular}{ll|l|l|l|l|l|}
$\mathrm{x} \times \mathrm{x} 0 \mathrm{x} \times \mathrm{x}$ & $c_{3,5}$ & $c_{2,4}$ & $c_{1,3}$ & $c_{0,2}$ & $c_{4,1}$ & $c_{3,0}$ Scan chain 3 \\
\hline
\end{tabular}

\begin{tabular}{ll|l|l|l|l|l|l}
$\mathrm{x} \times \mathrm{x}$ x 1 x x & $c_{4,5}$ & $c_{3,4}$ & $c_{2,3}$ & $c_{1,2}$ & $c_{0,1}$ & $c_{4,0}$ \\
\cline { 2 - 5 }
\end{tabular}

Fig. 5. An illustration of the reorganization of scan chains.

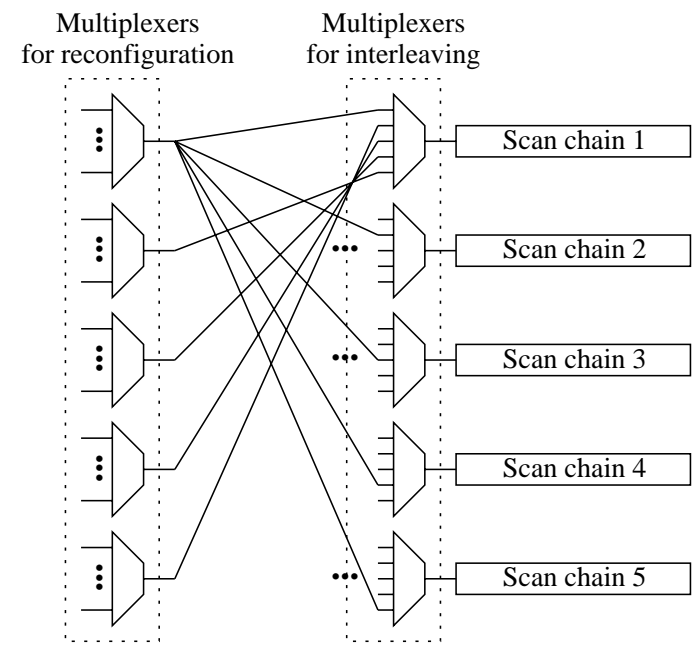

Fig. 6. An illustration of interleaving of the inputs of scan chains.

the connections between the two stages of multiplexers. For a CUT with $m$ scan chains, $m$ multiplexers are used for reconfiguration, and $m$ multiplexers are inserted for interleaving. Each of the multiplexers used for interleaving has $m$ inputs, which are selected in ascending order during the shifting in of a test pattern, i.e., the first input is selected for the first scan clock cycle, the second input is selected for the second scan clock cycle, and so on. After the $m$ th input is selected, the procedure is repeated with the first input. We use $A_{i}$ to denote the output the $i$ th multiplexers for reconfiguration and $B_{i, j}$ to denote the $j$ th input of the $i$ th multiplexers for interleaving, where $i, j=1,2, \ldots, m$. The interleaving is carried out by connecting the inputs of the multiplexers for interleaving with 
the outputs of multiplexers for reconfiguration such that

$$
B_{i, j}= \begin{cases}A_{i-j+1} & \text { if } i \geq j \\ A_{i-j+1+m} & \text { if } i<j .\end{cases}
$$

Consider the test cube shown in Fig. 5. After adding the second stage of multiplexers and connecting the inputs of the multiplexers for interleaving with the outputs of the multiplexers for reconfiguration, as shown in Fig. 6 (only the connections related to the first RIN multiplexer are shown for clarity), the output of the first multiplexer for reconfiguration should match with "xxxx1x", the same string as that in scan cell reorganization method. Note that the above reorganization and interleaving procedure yield the same set of test cubes.

\section{EXPERIMENTAL RESULTS}

In this section, we present experimental results for the seven largest ISCAS-89 circuits and for test cubes for the two production circuit from IBM. We use three sets of test cubes $T_{D}$ for the large ISCAS-89 circuits. The first set of test cubes are obtained from the Mintest ATPG program [9] without dynamic compaction, and by targeting all the irredundant single stuckat faults. The other two test sets are the same test sets used in [10]. The second set of the test cubes are obtained without an initial pseudo-random pattern application, and they target all irredundant faults. The third set of the test cubes for the random-pattern-resistant faults is obtained after 10000 pseudorandom patterns are applied to the circuits. In all the following experiments, we used a 64-bit primitive-polynomial LFSR with a fixed seed as the pseudo-random pattern generator, and we assumed that each of the ISCAS-89 circuits contains 32 scan chains. For simplicity of presentation, we assume that the circuits have balanced scan chains. When the scan chains are unbalanced, we can view them as being balanced through the addition of dummy scan cells.

Table I presents the results on test set embedding where $T_{D}$ is obtained using the Mintest program. We use a value of 5000 for the MaxSkipPatterns parameter for this set of experiments. The fifth column shows the total number of configurations needed to embed $T_{D}$. The total number of patterns applied to the circuit is listed in the sixth column. The testing time in clock cycles is obtained as the product of the total number of patterns and $(l+1)$, where $l$ is the length of scan chains. The hardware overhead includes the multiplexers for reconfiguration, the decoder and the configuration counter. We calculate the gate equivalent (GE) value for the hardware overhead using the method suggested in [28]: $0.5 n$ for an $n$-input NAND or NOR gate, and 0.5 for an inverter. We also use 0.5 as the GE value for a transmission gate, and a GE value of 4 for a flip-flop. The percentage hardware overhead is obtained from the ratio of the amount of BIST hardware in GEs to the GE count of the CUT. The pattern counter is not included in the calculation of the BIST hardware overhead because it is required for any scan-BIST scheme. The encoding efficiency shown in the last column is the ratio of the number of care bits in the test set to the amount of storage needed.

The results in Table I show that only a small number of control bits (at most a few hundred) are required for test set embedding. The hardware overhead of the RIN, the decoder, and the configuration counter are also very small, less then $8 \%$ for five circuits, and only $5.19 \%$ on average.

As indicated in Table I, we were unable to embed the test cubes for the $\mathrm{s} 38417$ benchmark circuit because the care bits in these test cubes are highly clustered. As a result, it is difficult to match these cubes to the patterns obtained from the LFSR. We therefore considered scan chain reorganization to obtain experimental results for s38417. Table II shows the result obtained with scan chain reorganization for the seven largest benchmark circuits with 32 scan chains each.

Tables III and IV present experimental results on the embedding of test cubes from [10] targeting all faults, without scan chain reorganization and with scan chain reorganization, respectively. In these two sets of experiments, we set the parameter MaxSkipPatterns to 10000. As indicated in Table III, we were unable to embed the test cubes of s38417 due to a high degree of clustering of its care bits. This problem was addressed using scan chain reorganization; the results are shown in Table IV. Scan chain reorganization for this set of test cubes reduces the number of configurations, and hence the storage and the hardware overhead for all circuits except s5378.

Table $\mathrm{V}$ presents experimental results obtained using the test cubes from [10] that target random-pattern-resistant faults. We assume that the RIN is bypassed using multiplexers for the first 10000 pseudo-random patterns. We considered scan chain reorganization for these experiments. As expected, compared with the results for test sets targeting all faults, the total number of patterns here is much smaller. Thus the testing time is also much less than in Table IV. The average storage requirement for all seven circuits is reduced from 900 bits to 534 bits.

In the above set of experiments, we assume that the information on the different number of patterns for each configuration is stored on-chip. If a fixed number of patterns is applied per configuration, then no storage is required. A tradeoff is that a fixed number of patterns per configuration might increase the number of configurations, and therefore increase the hardware overhead. In the next set of experiments, we set the number of patterns for each configuration to 1000. As expected, the results in Table VI show that the hardware overhead increase slightly for each circuit. Nevertheless and important benefit here is that no storage is necessary for control bits.

Table VII compares the storage requirements of the proposed approach with hybrid BIST based on weighted pseudorandom patterns [15], test vector encoding using partial LFSR reseeding [17], the BIST scheme based on reseeding of folding counter [10], and two-dimensional test data compression [20]. The results for the proposed approach are taken from Table V. All these methods rely on 10000 initial pseudo-random patterns to eliminate the easy to detect faults, except for [15], 
TABLE I

EXPERIMENTAL RESULTS FOR MiNTEST TEST SETS TARGETING ALL FAULTS.

\begin{tabular}{|c|c|c|c|c|c|c|c|c|c|}
\hline Circuit & $\begin{array}{l}\text { No. of } \\
\text { test } \\
\text { cubes }\end{array}$ & $\begin{array}{l}\text { No. of } \\
\text { scan } \\
\text { cells }\end{array}$ & $\begin{array}{l}\text { Length } \\
\text { of scan } \\
\text { chain }\end{array}$ & $\begin{array}{c}\text { No. of } \\
\text { configurations }\end{array}$ & $\begin{array}{c}\text { No. of } \\
\text { BIST } \\
\text { patterns }\end{array}$ & $\begin{array}{c}\text { Testing } \\
\text { time (clock } \\
\text { cycles) }\end{array}$ & $\begin{array}{c}\text { Hardware overhead } \\
\text { in GEs, and as a } \\
\text { percentage }\end{array}$ & $\begin{array}{c}\text { Storage } \\
\text { requirement } \\
\text { (bits) }\end{array}$ & $\begin{array}{l}\text { Encoding } \\
\text { efficiency }\end{array}$ \\
\hline s5378 & 1458 & 214 & 7 & 9 & 157184 & 1257472 & $184.5(7.08 \%)$ & 162 & 81.44 \\
\hline s9234 & 1928 & 247 & 8 & 34 & 363551 & 3271959 & $690.0(14.82 \%)$ & 646 & 39.57 \\
\hline s13207 & 3237 & 700 & 22 & 9 & 322526 & 7418098 & $184.5(2.36 \%)$ & 171 & 157.02 \\
\hline s15850 & 3920 & 611 & 20 & 24 & 379377 & 7966917 & $478.5(5.47 \%)$ & 456 & 67.02 \\
\hline s35932 & 10810 & 1763 & 56 & 4 & 19756 & 1126092 & $79.0(0.38 \%)$ & 60 & 771.18 \\
\hline s38417* & 10771 & 1664 & 52 & - & - & - & - & - & - \\
\hline s38584 & 13468 & 1464 & 46 & 11 & 377804 & 17756788 & $221.5(1.05 \%)$ & 209 & 441.15 \\
\hline Average & - & - & - & 15 & 270033 & 6466221 & $306.3(5.19 \%)$ & 284 & 259.56 \\
\hline
\end{tabular}

${ }^{*}$ Care bits are highly clustered hence test cubes could not be embedded with MaxSkipPatterns $=5000$.

TABLE II

EXPERIMENTAL RESULTS FOR MINTEST TEST SETS TARGETING ALL FAULTS AND WITH SCAN CELL REORGANIZATION.

\begin{tabular}{|c|c|c|c|c|c|c|c|c|c|}
\hline Circuit & $\begin{array}{l}\text { No. of } \\
\text { test } \\
\text { cubes }\end{array}$ & $\begin{array}{l}\text { No. of } \\
\text { scan } \\
\text { cells }\end{array}$ & $\begin{array}{l}\text { Length } \\
\text { of scan } \\
\text { chain }\end{array}$ & $\begin{array}{c}\text { No. of } \\
\text { configurations }\end{array}$ & $\begin{array}{c}\text { No. of } \\
\text { BIST } \\
\text { patterns }\end{array}$ & $\begin{array}{c}\text { Testing } \\
\text { time (clock } \\
\text { cycles) }\end{array}$ & $\begin{array}{c}\text { Hardware overhead } \\
\text { in GEs, and as a } \\
\text { percentage }\end{array}$ & $\begin{array}{c}\text { Storage } \\
\text { requirement } \\
\text { (bits) }\end{array}$ & $\begin{array}{l}\text { Encoding } \\
\text { efficiency }\end{array}$ \\
\hline $\mathrm{s} 5378$ & 1458 & 214 & 7 & 8 & 67988 & 543904 & $157.5(6.04 \%)$ & 136 & 97.01 \\
\hline s9234 & 1928 & 247 & 8 & 36 & 135765 & 1221885 & $729.0(15.66 \%)$ & 648 & 39.44 \\
\hline s13207 & 3237 & 700 & 22 & 9 & 152596 & 3509708 & $184.5(2.36 \%)$ & 162 & 165.75 \\
\hline s15850 & 3920 & 611 & 20 & 22 & 222336 & 4669056 & $440.5(5.04 \%)$ & 396 & 77.18 \\
\hline s35932 & 10810 & 1763 & 56 & 5 & 7079 & 403503 & $103.5(0.49 \%)$ & 65 & 711.86 \\
\hline s38417 & 10771 & 1664 & 52 & 272 & 625273 & 33139469 & $5752.5(26.16 \%)$ & 5440 & 26.14 \\
\hline s38584 & 13468 & 1464 & 46 & 12 & 383009 & 18001423 & $240.0(1.14 \%)$ & 228 & 404.39 \\
\hline Average & - & - & - & 52 & 227721 & 8784135 & $1086.8(8.13 \%)$ & 1011 & 217.39 \\
\hline
\end{tabular}

TABLE III

EXPERIMENTAL RESULTS FOR TEST SETS FROM [10] TARGETING ALL FAULTS.

\begin{tabular}{|c|c|c|c|c|c|c|c|c|c|}
\hline Circuit & $\begin{array}{l}\text { No. of } \\
\text { test } \\
\text { cubes }\end{array}$ & $\begin{array}{l}\text { No. of } \\
\text { scan } \\
\text { cells }\end{array}$ & $\begin{array}{l}\text { Length } \\
\text { of scan } \\
\text { chain }\end{array}$ & $\begin{array}{c}\text { No. of } \\
\text { configurations }\end{array}$ & $\begin{array}{l}\text { No. of } \\
\text { BIST } \\
\text { patterns }\end{array}$ & $\begin{array}{c}\text { Testing } \\
\text { time (clock } \\
\text { cycles) }\end{array}$ & $\begin{array}{c}\text { Hardware overhead } \\
\text { in GEs, and as a } \\
\text { percentage }\end{array}$ & $\begin{array}{l}\text { Storage } \\
\text { requirement } \\
\text { (bits) }\end{array}$ & $\begin{array}{l}\text { Encoding } \\
\text { efficiency }\end{array}$ \\
\hline s5378 & 4397 & 214 & 7 & 6 & 289754 & 2318032 & $121.5(4.66 \%)$ & 114 & 313.81 \\
\hline s9234 & 6475 & 247 & 8 & 57 & 394496 & 3550464 & $1138.5(24.45 \%)$ & 1083 & 72.55 \\
\hline s13207 & 9608 & 700 & 22 & 11 & 580521 & 13351983 & $221.5(2.83 \%)$ & 220 & 315.13 \\
\hline s15850 & 11330 & 611 & 20 & 32 & 758497 & 15928437 & $630.5(7.21 \%)$ & 640 & 129.96 \\
\hline s38417* & 30859 & 1664 & 52 & - & - & - & - & - & - \\
\hline s38584 & 34493 & 1464 & 46 & 12 & 526555 & 24748085 & $240.0(1.14 \%)$ & 240 & 822.90 \\
\hline Average & - & - & - & 24 & 509964 & 11979400 & $470.4(8.06 \%)$ & 459 & 330.87 \\
\hline
\end{tabular}

${ }^{*}$ Care bits are highly clustered hence test cubes could not be embedded with MaxSkipPatterns $=10000$.

TABLE IV

EXPERIMENTAL RESULTS FOR TEST SETS FROM [10] TARGETING ALL FAULTS AND WITH SCAN CELL REORGANIZATION.

\begin{tabular}{|c|c|c|c|c|c|c|c|c|c|}
\hline Circuit & $\begin{array}{l}\text { No. of } \\
\text { test } \\
\text { cubes }\end{array}$ & $\begin{array}{l}\text { No. of } \\
\text { scan } \\
\text { cells }\end{array}$ & $\begin{array}{l}\text { Length } \\
\text { of scan } \\
\text { chain }\end{array}$ & $\begin{array}{c}\text { No. of } \\
\text { configurations }\end{array}$ & $\begin{array}{c}\text { No. of } \\
\text { BIST } \\
\text { patterns }\end{array}$ & $\begin{array}{c}\text { Testing } \\
\text { time (clock } \\
\text { cycles) }\end{array}$ & $\begin{array}{c}\text { Hardware overhead } \\
\text { in GEs, and as a } \\
\text { percentage }\end{array}$ & $\begin{array}{c}\text { Storage } \\
\text { requirement } \\
\text { (bits) }\end{array}$ & $\begin{array}{l}\text { Encoding } \\
\text { efficiency }\end{array}$ \\
\hline s5378 & 4397 & 214 & 7 & 8 & 250275 & 2002200 & $157.5(6.04 \%)$ & 144 & 248.43 \\
\hline s9234 & 6475 & 247 & 8 & 31 & 562133 & 5059197 & $611.5(13.13 \%)$ & 620 & 126.73 \\
\hline s13207 & 9608 & 700 & 22 & 11 & 297713 & 6847399 & $221.5(2.83 \%)$ & 209 & 331.72 \\
\hline s15850 & 11330 & 611 & 20 & 18 & 634468 & 13323828 & $364.5(4.17 \%)$ & 360 & 231.04 \\
\hline s38417 & 30859 & 1664 & 52 & 184 & 1707485 & 90496705 & $3808.0(17.32 \%)$ & 3864 & 75.35 \\
\hline s38584 & 34493 & 1464 & 46 & 10 & 736686 & 34624242 & $203.0(0.96 \%)$ & 200 & 987.48 \\
\hline Average & - & - & - & 44 & 698126 & 25392262 & $894.3(7.41 \%)$ & 900 & 333.46 \\
\hline
\end{tabular}

which uses 32000 pseudo-random patterns. The results show that in all but one case, the proposed approach requires less storage than other methods. (The hybrid BIST method [15] requires less storage for s9234.)

In Table VIII, we compare the proposed method with scan- based 3-weight weighted random BIST [30]. Since no storage of seeds or control bits is required in [30], we use the results from Table VI for comparison. The third column of Table VIII lists the number of pseudo-random patterns required to achieve $100 \%$ coverage of detectable single stuck-at faults, as reported

Paper 17.3 
TABLE V

EXPERIMENTAL RESULTS FOR TEST SETS FROM [10] TARGETING RANDOM-PATTERN-RESISTANT FAULTS WITH SCAN CHAIN REORGANIZATION.

\begin{tabular}{|c|c|c|c|c|c|c|c|c|c|}
\hline Circuit & $\begin{array}{c}\text { No. of } \\
\text { test } \\
\text { cubes }\end{array}$ & $\begin{array}{c}\text { No. of } \\
\text { scan } \\
\text { cells }\end{array}$ & $\begin{array}{c}\text { Length } \\
\text { of scan } \\
\text { chain }\end{array}$ & $\begin{array}{c}\text { No. of } \\
\text { configurations }\end{array}$ & $\begin{array}{c}\text { No. of } \\
\text { BIST } \\
\text { patterns }\end{array}$ & $\begin{array}{c}\text { Testing } \\
\text { time (clock } \\
\text { cycles) }\end{array}$ & $\begin{array}{c}\text { Hardware overhead } \\
\text { in GEs, and as a } \\
\text { percentage }\end{array}$ & $\begin{array}{c}\text { Storage } \\
\text { requirement } \\
\text { (bits) }\end{array}$ & $\begin{array}{c}\text { Encoding } \\
\text { efficiency }\end{array}$ \\
\hline s5378 & 39 & 214 & 7 & 5 & 3269 & 26152 & $103.5(3.97 \%)$ & 60 & 10.27 \\
\hline s9234 & 698 & 247 & 8 & 34 & 34341 & 309069 & $690.0(14.82 \%)$ & 544 & 32.53 \\
\hline s13207 & 556 & 700 & 22 & 5 & 54776 & 1259848 & $103.5(1.32 \%)$ & 80 & 106.71 \\
\hline s15850 & 654 & 611 & 20 & 22 & 26968 & 566328 & $440.5(5.04 \%)$ & 330 & 45.15 \\
\hline s38417 & 2219 & 1664 & 52 & 120 & 103653 & 5493609 & $2431.5(11.06 \%)$ & 2040 & 28.66 \\
\hline s38584 & 441 & 1464 & 46 & 10 & 21282 & 1000254 & $203.0(0.96 \%)$ & 150 & 48.74 \\
\hline Average & - & - & - & 33 & 40714 & 1442543 & $662.0(6.20 \%)$ & 534 & 45.34 \\
\hline
\end{tabular}

TABLE VI

EXPERIMENTAL RESULTS FOR TEST SETS FROM [10] TARGETING RANDOM-PATTERN-RESISTANT FAULTS WITH SCAN CELL REORGANIZATION AND A FIXED NUMBER OF PATTERNS PER CONFIGURATION.

\begin{tabular}{|c|c|c|c|c|c|c|c|c|}
\hline Circuit & $\begin{array}{c}\text { No. of } \\
\text { test } \\
\text { cubes }\end{array}$ & $\begin{array}{c}\text { No. of } \\
\text { scan } \\
\text { cells }\end{array}$ & $\begin{array}{c}\text { Length } \\
\text { of scan } \\
\text { chain }\end{array}$ & $\begin{array}{c}\text { No. of } \\
\text { configurations }\end{array}$ & $\begin{array}{c}\text { No. of } \\
\text { BIST } \\
\text { patterns }\end{array}$ & $\begin{array}{c}\text { Testing } \\
\text { time (clock } \\
\text { cycles) }\end{array}$ & $\begin{array}{c}\text { Hardware overhead } \\
\text { in GEs, and as a } \\
\text { percentage }\end{array}$ & $\begin{array}{c}\text { Storage } \\
\text { requirement } \\
\text { (bits) }\end{array}$ \\
\hline s5378 & 39 & 214 & 7 & 5 & 4003 & 32024 & $103.5(3.97 \%)$ & 0 \\
\hline s9234 & 698 & 247 & 8 & 37 & 36001 & 324009 & $748.5(16.08 \%)$ & 0 \\
\hline s13207 & 556 & 700 & 22 & 8 & 8000 & 184000 & $157.5(2.02 \%)$ & 0 \\
\hline s15850 & 654 & 611 & 20 & 24 & 23002 & 483042 & $478.5(5.47 \%)$ & 0 \\
\hline s38417 & 2219 & 1664 & 52 & 129 & 128001 & 6784053 & $2680.5(12.19 \%)$ & 0 \\
\hline s38584 & 441 & 1464 & 46 & 12 & 11001 & 517047 & $240(1.14 \%)$ & 0 \\
\hline Average & - & - & - & 36 & 35001 & 1387363 & $734.8(6.81 \%)$ & 0 \\
\hline
\end{tabular}

TABLE VII

COMPARISON OF STORAGE REQUIRED FOR VARIOUS BIST METHODS.

\begin{tabular}{|c|c|c|c|c|c|}
\hline Circuit & $\begin{array}{c}\text { Hybrid } \\
\text { BIST [15] }\end{array}$ & $\begin{array}{c}\text { Partial } \\
\text { Reseeding [17] }\end{array}$ & $\begin{array}{c}\text { Reseeding of } \\
\text { folding counter [10] }\end{array}$ & $\begin{array}{c}\text { Two-dimensional } \\
\text { compression [20] }\end{array}$ & $\begin{array}{c}\text { Proposed } \\
\text { approach }\end{array}$ \\
\hline s5378 & N/A & 502 & 132 & 196 & 60 \\
\hline s9234 & 371 & 5013 & 2310 & 3800 & 544 \\
\hline s13207 & 110 & 3008 & 247 & 1044 & 80 \\
\hline s15850 & 535 & 5204 & 2403 & 3360 & 330 \\
\hline s38417 & 2663 & 24513 & 6802 & 11214 & 2040 \\
\hline s38584 & 615 & 2942 & 660 & 2891 & 150 \\
\hline
\end{tabular}

TABLE VIII

COMPARISON WITH 3-WEIGHT WEIGHTED RANDOM BIST.

\begin{tabular}{|c|c|c|c|c|c|c|c|c|c|c|c|}
\hline \multirow[b]{2}{*}{ Circuit } & \multirow[b]{2}{*}{$\begin{array}{l}\text { No. of } \\
\text { scan } \\
\text { cells }\end{array}$} & \multirow[b]{2}{*}{$\begin{array}{c}\text { Required } \\
\text { random } \\
\text { patterns }\end{array}$} & \multicolumn{6}{|c|}{ 3-weight weighted random BIST [30] } & \multicolumn{3}{|c|}{ Proposed approach } \\
\hline & & & $\begin{array}{l}\text { No. of } \\
\text { random } \\
\text { patterns }\end{array}$ & $\begin{array}{c}\text { No. of } \\
\text { weighted } \\
\text { patterns }\end{array}$ & $\begin{array}{c}\text { Total } \\
\text { no. of } \\
\text { patterns }\end{array}$ & $\begin{array}{c}\text { Testing } \\
\text { time } \\
(\mathrm{ms})\end{array}$ & $\begin{array}{l}\text { Hardware } \\
\text { overhead } \\
\text { (parallel) }\end{array}$ & $\begin{array}{c}\text { Hardware } \\
\text { overhead } \\
\text { (serial) }\end{array}$ & $\begin{array}{c}\text { Total } \\
\text { no. of } \\
\text { patterns }\end{array}$ & $\begin{array}{c}\text { Testing } \\
\text { time } \\
\text { (ms) }\end{array}$ & $\begin{array}{c}\text { Hardware } \\
\text { overhead }\end{array}$ \\
\hline s5378 & 214 & $>10 \mathrm{M}$ & 4000 & 5120 & 9120 & 98.04 & 16 & 86.5 & 14003 & 5.60 & 103.5 \\
\hline s9234 & 247 & $11 \mathrm{M}$ & 32000 & 11264 & 43264 & 536.47 & 91 & 146.5 & 46001 & 20.70 & 748.5 \\
\hline s13207 & 700 & $264 \mathrm{~K}$ & 64000 & 6144 & 70144 & 2458.55 & 16.5 & 120.0 & 18000 & 20.70 & 157.5 \\
\hline s15850 & 611 & $>100 \mathrm{M}$ & 64000 & 21504 & 85504 & 2616.42 & 82 & 264.5 & 33002 & 34.65 & 478.5 \\
\hline s38417 & 1664 & $>100 \mathrm{M}$ & 32000 & 53248 & 85248 & 7096.90 & 169.5 & 626.6 & 138001 & 365.70 & 2680.5 \\
\hline s38584 & 1464 & $>100 \mathrm{M}$ & 2000 & 16384 & 18384 & 1346.63 & 30 & 197.0 & 21001 & 49.35 & 240 \\
\hline
\end{tabular}

in $[1,30]$. The total number of patterns listed for our approach is obtained by adding 10000 to the number of patterns listed in Table VI. The testing times listed in the table are obtained by assuming a $20 \mathrm{MHz}$ scan clock frequency. The testing time for the proposed approach is less, even though [30] requires a smaller number of patterns for some circuits. This is because we use a multiple scan chain architecture, whereas [30] is based on a single scan chain architecture. The use of a sin- gle scan chain architecture ensures that the hardware overhead in [30] is lower; however, in order to scale 3-weight weighted random BIST to multiple scan chains, separate decoding logic is needed for each scan chain, which contributes to increased hardware overhead. The parallel scheme in [30] requires even less hardware, but it relies on explicit control of the set and reset signal of the flip-flops after scan cell reordering.

In order to evaluate the effectiveness of the proposed ap- 
TABLE IX

RESULTS FOR TEST CUBES FOR CIRCUITS FROM IBM.

\begin{tabular}{|c|c|c|c|c|c|c|c|c|c|}
\hline Circuit & $\begin{array}{l}\text { No. of } \\
\text { test } \\
\text { cubes }\end{array}$ & $\begin{array}{l}\text { No. of } \\
\text { scan } \\
\text { cells }\end{array}$ & $\begin{array}{l}\text { No. of } \\
\text { scan } \\
\text { chains }\end{array}$ & $\begin{array}{l}\text { Length } \\
\text { of scan } \\
\text { chain }\end{array}$ & $\begin{array}{c}\text { No. of } \\
\text { configurations }\end{array}$ & $\begin{array}{c}\text { Testing } \\
\text { time (clock } \\
\text { cycles) }\end{array}$ & $\begin{array}{c}\text { Hardware overhead } \\
\text { in GEs, and as a } \\
\text { percentage }\end{array}$ & $\begin{array}{l}\text { Storage } \\
\text { requirement } \\
\text { (bits) }\end{array}$ & $\begin{array}{l}\text { Encoding } \\
\text { efficiency }\end{array}$ \\
\hline \multirow{3}{*}{ CKT1 } & \multirow{3}{*}{32} & \multirow{3}{*}{362921} & 64 & 5671 & 258 & 181504 & $9586.5(0.15 \%)$ & 3096 & 23.89 \\
\hline & & & 128 & 2836 & 177 & 90784 & $12160.5(0.19 \%)$ & 2124 & 34.82 \\
\hline & & & 256 & 1418 & 437 & 45408 & $58161.5(0.89 \%)$ & 4807 & 15.39 \\
\hline \multirow{3}{*}{ CKT2 } & \multirow{3}{*}{4} & \multirow{3}{*}{1031072} & 64 & 16111 & 277 & 64448 & $10289.5(0.77 \%)$ & 3047 & 28.52 \\
\hline & & & 128 & 8056 & 166 & 32228 & $11407.0(0.86 \%)$ & 1660 & 52.35 \\
\hline & & & 256 & 4028 & 516 & 16116 & $68931.0(5.19 \%)$ & 4644 & 18.71 \\
\hline
\end{tabular}

proach for large circuits, we applied the method to two real-life test sets from industry. The test set provided to us for the first circuit (CKT1) from IBM consists of 32 statically-compacted scan vectors (a total of 362921 bits of test data per vector). This microprocessor design consists of 3.6 million gates and 726000 latches. The test set for a second microprocessor circuit (CKT2) from IBM consists of a set of 4 scan vectors (a total of 1031072 bits of test data per vector); this design contains 1.2 million gates and 32200 latches. Since we do not have access to the gate-level models for these circuits, we are unable to report fault coverage values for these test sets. The number of scan chains is varied from 64 to 256 for these two circuits. We modified the simulation procedure such that the configuration of the interconnection network can be changed during the application of a test cube, and we set the parameter MaxSkipPatterns to 0. Accordingly, in the proposed BIST architecture shown in Fig. 2(a), the stored control bits are the number of bits per configuration instead of the number of patterns per configuration, and the pattern counter is replaced by a bit counter which counts the number of bits that have been shifted into the scan chains. Table IX lists the results for these two industrial circuits. Since no additional pseudo-random patterns are applied to these circuits, the testing time is simply equal to the number of the clock cycles that are required to shift the deterministic patterns into the scan chains, and capture and scan out the responses. The hardware overhead is negligible and very high encoding efficiency is achieved for both circuits. Using a Sun-Blade-1000 workstation with one $750 \mathrm{MHz}$ UltraSPARC-III CPU and 1GB memory, the results for each row of Table IX are obtained in less than two minutes.

\section{CONCLUSION}

We have presented a new approach for deterministic BIST based on the use of a reconfigurable interconnection network (RIN). The RIN is placed between the outputs of pseudorandom pattern generator, e.g., an LFSR, and the scan inputs of the circuit under test (CUT). It consists only of multiplexer switches and it is designed using a synthesis procedure that takes as inputs the pseudo-random sequence from the LFSR and the deterministic test cubes for the CUT. The connections between the LFSR and the scan chains can be changed dynamically (reconfigured) during a test session. In this way, the RIN is used to match the LFSR outputs to the set of test cubes $T_{D}$. The control data bits used for reconfiguration guarantee that $T_{D}$ is embedded in the test patterns applied to the CUT. We have shown through several sets of experiments that the proposed approach requires very little hardware overhead. We have also shown that the fewer control bits are required compared to the storage required for reseeding methods or for hybrid BIST. Finally, as a non-intrusive BIST solution, the proposed approach does not require any circuit redesign and it has minimal impact on circuit performance.

We are currently extending this work to ensure that undesirable input patterns that cause problems such as bus contention are forwarded to the scan chains by the RIN. It appears that this problem, which is typical of most logic BIST techniques, can be handled by suitably modifying the RIN synthesis procedure.

\section{ACKNOWLEDGMENT}

We thank Prof. Sybille Hellebrand of University of Innsbruck in Austria for providing us with the test cubes used in [10]. We also thank Brion Keller of Cadence Design Systems (formerly with IBM Corporation) for providing us with the test cubes for the production circuits from IBM.

\section{REFERENCES}

[1] M. F. AlShaibi and C. R. Kime, "MFBIST: a BIST method for random pattern resistant circuits," Proc. Int. Test Conf., pp. 176-185, 1996.

[2] C. Barnhart, V. Brunkhorst, F. Distler, O. Farnsworth, B.Keller and B. Koenemann, "OPMISR: the foundation for compressed ATPG vectors,” Proc. Int. Test Conf., pp. 748-757, 2001.

[3] I. Bayraktaroglu and A. Orailoglu, "Test volume and application time reduction through scan chain concealment," Proc. ACM/IEEE Design Automation Conf., pp. 151-155, 2001.

[4] A. Chandra and K. Chakrabarty, "Frequency-directed run-length (FDR) codes with application to system-on-a-chip test data compression," Proc. VLSI Test Symp., pp. 42-47, 2001.

[5] A. Chandra and K. Chakrabarty, "System-on-a-chip test data compression and decompression architectures based on Golomb codes," IEEE Trans. Computer-Aided Design, vol. 20, pp. 355368, March 2001.

[6] K.-T. Cheng and C.-J. Lin, "Timing driven test point insertion for full-scan and partial-scan BIST," Proc. Intl. Test Conf., pp. 506-514, 1995. 
[7] A. El-Maleh, S. al Zahir and E. Khan, "A geometric-primitivesbased compression scheme for testing systems-on-chip," Proc. VLSI Test Symp., pp. 54-59, 2001.

[8] P. T. Gonciari, B. Al-Hashimi and N. Nicolici, "Improving compression ratio, area overhead, and test application time for system-on-a-chip test data compression/decompression," Proc. Design, Automation and Test in Europe Conf., pp. 604-611, 2002.

[9] I. Hamzaoglu and J. H. Patel, "Test set compaction algorithms for combinational circuits," Proc. Int. Conf. CAD, pp. 283-289, 1998.

[10] S. Hellebrand, H.-G. Liang and H.-J. Wunderlich, "A mixedmode BIST scheme based on reseeding of folding counters," Proc. Int. Test Conf., pp. 778-784, 2000.

[11] G. Hetherington, T. Fryars, N. Tamarapalli, M. Kassab, A. Hassan and J. Rajski, "Logic BIST for large industrial designs: real issues and case studies," Proc. Int. Test Conf., pp. 358-367, 1999.

[12] V. Iyengar, K. Chakrabarty and B. T. Murray, "Deterministic built-in pattern generation for sequential circuits," Journal of Electrinic Testing: Theory and Applications, vol. 15, pp. 97115,1999

[13] A. Jas and N. A. Touba, "Test vector decompression via cyclical scan chains and its application to testing core-based design," Proc. Int. Test Conf., pp. 458-464, 1998.

[14] A. Jas, J. Ghosh-Dastidar and N. A. Touba, "Scan vector compression/decompression using statistical coding," Proc. VLSI Test Symp., pp. 114-120, 1999.

[15] A. Jas, C. V. Krishna and N. A. Touba, "Hybrid BIST based on weighted pseudo-random testing: a new test resource partitioning scheme," Proc. VLSI Test Symp., pp. 2-8, 2001.

[16] B. Koenemann, C. Barnhart, B.Keller, T. Snethen, O. Farnsworth and D. Wheater, "A SmartBIST variant with guaranteed encoding," Proc. Asian Test Symp., pp. 325-330, 2001.

[17] C. V. Krishna, A. Jas and N. A. Touba, "Test vector encoding using partial LFSR reseeding," Proc. Int. Test Conf., pp. 885893, 2001.

[18] C. V. Krishna and N. A. Touba, "Reducing test data volume using LFSR reseeding with seed compression," Proc. Int. Test Conf., pp. 321-330, 2002.

[19] L. Li and K. Chakrabarty, "Test data compression using dictionaries with fixed-length indices," Proc. VLSI Test Symp., pp. 219-224, 2003.

[20] H.-G. Liang, S. Hellebrand and H.-J. Wunderlich, "Twodimensional test data compression for scan-based deterministic BIST," Proc. Int. Test Conf., pp. 894-902, 2001.

[21] M. Nakao, S. Kobayashi, K. Hatayama, K. Iijima and S. Terada, "Low overhead test point insertion for scan-based BIST," Proc. Int. Test Conf., pp. 348-357, 1999.

[22] J. Rajski, J. Tyszer and N. Zacharia, "Test data decompression for multiple scan designs with boundary scan," IEEE Trans. Computers, vol. 47, pp. 1188-1200, November 1998.

[23] J. Rajski, J. Tyszer, M. Kassab, N. Mukherjee, R. Thompson, H. Tsai, A. Hertwig, N. Tamarapalli, G. Mrugalski, G. Eide and J. Qian, "Embedded deterministic test for low-cost manufacturing test," Proc. Int. Test Conf., pp. 301-310, 2002.

[24] S. Reda and A. Orailoglu, "Reducing test application time through test data mutation encoding," Proc. Design, Automation and Test in Europe Conf., pp. 387-393, 2002.

[25] S. M. Reddy, K. Miyase, S. Kajihara and I. Pomeranz, "On test data volume reduction for multiple scan chain design," Proc. VLSI Test Symp., pp. 103-108, 2002.

[26] L. Schafer, R. Dorsch and H.-J. Wunderlich, "RESPIN++ deterministic embedded test," Proc. European Test Workshop, pp. 37-44, 2002.

[27] C. Schotten and H. Meyr, "Test point insertion for an area efficient BIST,” Proc. Intl. Test Conf., pp. 515-523, 1995.

[28] N. A. Touba and E. J. McCluskey, "Altering a pseudo-random bit sequence for scan based BIST," Proc. Int. Test Conf., pp. 167-175, 1996.

[29] E. H. Volkerink, A. Khoche and S. Mitra, "Packet-based input test data compression techniques," Proc. Int. Test Conf., pp. 154-163, 2002.

[30] S. Wang, "Low hardware overhead scan based 3-weight weighted random BIST,' Proc. Int. Test Conf., pp. 868-877, 2001.

[31] H.-J. Wunderlich and G. Kiefer, "Bit-flipping BIST," Proc. Int. Conf. Computer-Aided Design, pp. 337-343, 1996. 